\title{
El computador como determinante del acceso a Internet en hogares de la República Dominicana
}

\author{
Por Fabricio Gómez Mazara*
}

Recibido: 1/7/2017 - Aprobado: 15/8/2017

\begin{abstract}
Resumen
La literatura relevante sobre los impactos del uso de Internet en el bienestar de los hogares ha identificado dos problemas centrales: el primero, denominado "doble causalidad" o "causalidad simultánea", parte del principio de que el uso de Internet estaría determinado por un conjunto de indicadores de bienestar, tales como el ingreso familiar y el nivel educativo del jefe o de la jefa de hogar. En este trabajo se utilizó un modelo econométrico de probabilidad respecto de hogares que tienen o no computador y acceso a Internet. Los resultados confirman que variables como el ingreso, la educación y las zonas rurales / urbanas son determinantes claves de la difusión de las TIC.
\end{abstract}

Palabras clave: hogares; computador y acceso a Internet.

Códigos del JEL: C19, C30, D10

* Escuela de Economía del Instituto Tecnológico de Santo Domingo (INTEC) e Instituto Dominicano de las Telecomunicaciones (INDOTEL). Dirección: Consejo Directivo, INDOTEL, Apartado Postal 10148. Avenida Abraham Lincoln No. 962. Santo Domingo, República Dominicana. T: 829732 5555. (e-mail: fgomez@intec.edu.do). Agradecimientos: agradezco los comentarios de Ana Carolina Franco, Luis Scheker y Amparo Arango, así como a la Dirección Ejecutiva y la Presidencia del Consejo Directivo del INDOTEL. 


\title{
The computer as a determinant of Internet access for households in the Dominican Republic
}

\author{
By FABricio Gómez MaZARA*
}

Submitted: 7/1/2017 - Accepted: 8/15/2017

\begin{abstract}
The relevant literature on the impacts of Internet use on household welfare has identified two central problems. The first, called "double causality" or "simultaneous causation", starts from the principle that the use of the Internet would be determined by a set of welfare indicators such as: family income and educational level of the head of household. This paper used an econometric model of probability that a household has or has not adopted computers and Internet access. The results confirm that variables such as income, education and rural urban areas are key determinants of Information and Communication Technologies (ICT) diffusion.
\end{abstract}

Keywords: household; computer and Internet access.

JEL Classification codes: C19, C30, D10

* Department of Economics, Instituto Tecnológico de Santo Domingo (INTEC) and Dominican Institute of Telecommunications (INDOTEL). Address: Board of Directors. Postal Mail 10148. Abraham Lincoln Ave. No. 962. Santo Domingo, Dominican Republic. T: 829 732 5555. (e-mail: fgomez@intec.edu.do). Acknowledgements: I would like to thank Ana Carolina Franco, Luis Scheker and Amparo Arango, as well as the Executive Directorate and the Chairman of the Directing Council of INDOTEL. 


\section{Introducción}

La interrelación de la demanda de acceso a Internet y la disponibilidad de computadoras en el hogar toma relevancia para el diseño de las políticas públicas en términos regulatorios debido a la necesidad de orientar programas que garanticen una mayor cobertura en el segmento residencial, así como de acceso universal para la puesta en marcha de proyectos en zonas rurales y marginales.

El Internet ha transformado las relaciones familiares, sociales, laborales, productivas y entre instituciones de diversa naturaleza en las sociedades, representando un elemento esencial para el bienestar de los hogares. Desde el punto de vista cultural, ha modificado los hábitos personales cambiando la percepción que tienen las personas del mundo y de sí mismas.

Frente al actual escenario mundial de concentración y exclusión digital, ampliar la cobertura de los hogares a las Tecnologías de la Información y la Comunicación (TIC) podría generar oportunidades para achicar la brecha entre aquellos "incluidos" y los "excluidos", de modo que todos puedan tener acceso al crecimiento y al desarrollo sustentable.

En tal sentido, la democratización de las TIC requiere una atención cuidadosa por parte de los gobiernos y, especialmente, del sistema educativo. Aun cuando la mayoría de los países están encarando acciones para integrarlas en la vida escolar y familiar, se trata de un proceso complejo cuando se toma en cuenta los importantes desafíos que rodean este tópico.

La evidencia empírica muestra el impacto positivo del acceso y uso del Internet sobre el capital humano, la productividad y el crecimiento económico. Si se considera que el Internet es una fuente de conocimiento e información, este tendría la capacidad de fortalecer el capital humano de los usuarios. 
Existe evidencia macroeconómica que sustenta que el acceso y uso intensivo de las TIC han sido elementos claves para el crecimiento y desarrollo económico, así como para el aumento de la productividad sectorial.

El acceso y uso intensivo de esta herramienta tecnológica abre un canal para el aumento de la productividad, eficiencia y diversificación de la producción en los usuarios, lo que permite lograr mejoras en el bienestar de los hogares. La disponibilidad de este servicio aumenta el consumo, principalmente en los hogares con menores recursos. Empero, sería difícil discernir si es que el uso de Internet genera mayores ingresos o si, por el contrario, los mayores ingresos incentivan la adopción de este servicio final de telecomunicaciones.

Sin embargo, para acceder a Internet es necesario disponer de un computador, ya sea en casa, en el trabajo o en la escuela. En tal sentido, existe un número importante de estudios que encuentran que la educación y el ingreso son determinantes tanto para la tenencia de computadores como para el uso de internet. Por otro lado, la ubicación geográfica del hogar es determinante para el uso de este servicio, dadas las condiciones de accesibilidad al servicio y el costo de adquirirlo.

La literatura relevante sobre los impactos del uso de Internet en el bienestar de los hogares ha identificado dos problemas centrales. El primero, denominado "doble causalidad" o "causalidad simultánea", parte del principio de que el uso de Internet estaría determinado por un conjunto de indicadores de bienestar, tales como el ingreso familiar y el nivel educativo del jefe o de la jefa de hogar.

El segundo problema está relacionado con variables no observables que pueden afectar la decisión del uso de Internet y del ingreso, tales como la preferencia sobre otros servicios, el coeficiente intelectual y la valoración otorgada por el hogar. 
En septiembre de 2016, el Poder Ejecutivo puso en marcha el programa República Digital que incluye un conjunto de políticas y acciones para promover la inclusión de las TIC en los procesos productivos, educativos, gubernamentales y de servicios a los ciudadanos.

El programa contempla cuatro componentes fundamentales: educación, acceso, productividad y empleo, así como el gobierno digital abierto. Para cumplir con los objetivos del programa, se crea la Comisión Presidencial de República Digital como el espacio institucional designando para el diseño, la implementación y el monitoreo del mismo.

La Comisión Presidencial de República Digital depende del Ministerio de la Presidencia y está conformada, además de este ministerio, por la Vicepresidencia de la República, el Instituto Dominicano de las Telecomunicaciones (INDOTEL), la Oficina Presidencial de Tecnologías de la Información y la Comunicación (OPTIC), la empresa de Transmisión Eléctrica Dominicana (ETED), así como los ministerios de Industria y Comercio (MIC), de Educación, Educación Superior, Ciencia y Tecnología (MESCYT) y Administración Pública (MAP).

Para el caso dominicano, la importancia de conocer la interrelación del acceso a Internet y la disponibilidad de computador en los hogares es indiscutible. A pesar de ello, su estudio presenta serias dificultades, entre las que ocupa un lugar destacado la carencia de información a nivel desagregado.

En la actualidad, la información disponible sobre este tema es confiable hasta cierto punto. Pero, la misma resulta insuficiente ya que solo se dispone de estadísticas a nivel agregado y es un tanto difícil aproximar el comportamiento de sus componentes. 
Este estudio fue realizado tomando como base el módulo de telecomunicaciones de la Encuesta de Suministro Eléctrico a Hogares (ESEH), de 2015, aplicada por muestreo a 5,810 hogares. Tiene representación a nivel de las 10 regiones de planificación del país y, por agregación, a nivel nacional.

Esta investigación pretende servir de insumo a los distintos actores del Programa República Digital permitiendo que estos puedan tener acceso a información actualizada y desagregada sobre cómo afecta la disponibilidad de computador en el hogar para poder acceder al Internet, dada la estructura actual de penetración de este servicio y el gasto en que incurren las familias.

Finalmente, queremos agradecer al Banco Interamericano de Desarrollo (BID) y al Instituto Tecnológico de Santo Domingo (INTEC) por haber cedido la base de datos de la Encuesta de Suministro Eléctrico en Hogares (ESEH) para la realización de este estudio.

\section{Aspectos metodológicos}

\section{A. Descripción}

El presente estudio es de tipo cuantitativo y fue abordado mediante un análisis descriptivo, con datos provenientes de fuentes secundarias y primarias.

Las fuentes secundarias utilizadas incluyen las encuestas sobre ingresos y gastos de los hogares, que son de dominio público. Las fuentes primarias incluyen la aplicación de una encuesta por muestreo dirigida a los hogares del país. También, en una segunda fase de la investigación, se obtuvo datos provenientes de una encuesta por muestreo dirigida a empresas en toda la geografía nacional. 


\section{B. Encuesta de Suministro Eléctrico en Hogares (ESEH)}

Se aplicó una encuesta por muestreo dirigida a los hogares del país para conocer aspectos específicos sobre el suministro de energía eléctrica y su impacto en las actividades socioeconómicas dentro del hogar. La encuesta tiene representación a nivel de las 10 regiones de planificación del país y, por agregación, a nivel nacional.

\section{Marco muestral}

La ESEH fue diseñada, seleccionada y levantada haciendo uso del marco muestral cartográfico digital elaborado por la Oficina Nacional de Estadística (ONE). El informante fue el jefe o la jefa del hogar, a quien se le aplicó un cuestionario de manera presencial.

El diseño muestral comprende muestras independientes para las 10 regiones de planificación, de acuerdo al decreto 710-04. En este, las unidades primarias de muestreo (UPM) fueron los segmentos censales y las unidades secundarias de muestreo (USM) fueron los hogares.

A continuación, se presenta un cuadro conteniendo el número de hogares por zona de residencia y por regiones.

Tabla 1. Número de hogares en República Dominicana distribuidos POR zONAS de RESIDENCIA, SEGÚN REGIONES

\begin{tabular}{|c|c|c|c|c|}
\hline \multirow{2}{*}{ Regiones } & \multicolumn{2}{|c|}{ Zonas } & \multirow{2}{*}{ Total } & \multirow{2}{*}{ Porcentaje urbano } \\
\hline & Urbana & Rural & & \\
\hline Cibao Norte & 301,096 & 201,074 & 502,170 & 59.96 \\
\hline Cibao Sur & 114,889 & 118,764 & 233,653 & 49.17 \\
\hline Cibao Nordeste & 102,681 & 118,325 & 221,006 & 46.46 \\
\hline Cibao Noroeste & 77,434 & 60,406 & 137,840 & 56.18 \\
\hline Valdesia & 162,521 & 147,848 & 310,369 & 52.36 \\
\hline Enriquillo & 70,083 & 33,956 & 104,039 & 67.36 \\
\hline El Valle & 44,515 & 54,633 & 99,148 & 44.90 \\
\hline Yuma & 124,702 & 53,153 & 177,855 & 70.11 \\
\hline Higuamo & 130,546 & 67,689 & 198,235 & 65.85 \\
\hline Ozama o Metropolitana & 829,191 & 82,297 & 911,488 & 90.97 \\
\hline Total & $1,957,658$ & 938,145 & $2,895,803$ & 67.60 \\
\hline
\end{tabular}

Fuente. Elaborado con datos de la ENFT 2013 del Banco Central de la República Dominicana. 


\section{Tamaño de la muestra}

Para el cálculo del tamaño de la muestra se trabajó con una confianza de $95 \%$ y un error muestral global de $0.8 \%$ y, a nivel de las regiones, se toleró un error muestral máximo que se ubica en el intervalo de $1.8 \%$ a $3.2 \%$.

Se tomó como variable de diseño la cobertura de la red eléctrica a nivel nacional, la cual se estimaba para el año 2013 en un $96.0 \%{ }^{1}$, y se trabajó bajo el supuesto de que el efecto del diseño no sería superior a 2.5, por aplicarse un muestreo bietápico.

El procedimiento estadístico utilizado para el cálculo de la muestra fue el aplicado para proporciones, en presencia de un muestreo bietápico y estratificado:

$$
\mathrm{n}_{0}=\frac{\mathrm{PQZ}^{2}}{\mathrm{E}^{2}}
$$

Este procedimiento es aplicado a poblaciones infinitas, pero como se tiene el número de hogares objeto de estudio, se hace el ajuste por finitud y luego se multiplica por el efecto del diseño:

$$
\mathrm{n}=\left[\frac{\mathrm{n}_{0}}{1+\left(\frac{\mathrm{n}_{0}}{\mathrm{~N}}\right)}\right] . \text { EFD }
$$

Donde:

n0: tamaño de la muestra

n: tamaño de muestra ajustado

P: proporción de la población que posee una cualquiera de las características a medir en el estudio

Q: proporción de la población que no posee una cualquiera de las características consideradas en el estudio

\footnotetext{
${ }^{1}$ Estimación promedio anual de la Encuesta Nacional de Fuerza de Trabajo, del Banco Central de la República Dominicana.
} 
Z2: nivel de confianza al cuadrado

E2: error muestral al cuadrado

EFD: efecto del diseño

La muestra calculada para el nivel nacional fue de 5,757 hogares, la cual fue afijada a las regiones y las zonas urbana y rural de forma proporcional a la raíz cuadrada del número de hogares existente en cada celda del cruce regiones x zonas.

Se trabajó con una sub-muestra fija de hogares en cada UPM a razón de cinco (5) hogares por cada unidad, resultando un total de 1,152 UPM.

Dado que la muestra se asignó entre las regiones y las zonas urbana y rural de forma desproporcionada al número de hogares existentes, debido a la transformación matemática aplicada al número de hogares (raíz cuadrada), se introdujeron factores de ponderación y expansión de la muestra para asegurar la adecuada inferencia estadística con los parámetros poblacionales, a nivel de cada una de las regiones del país.

A continuación, la tabla 2 presenta un cuadro con la muestra total, por regiones y por zonas urbanas y rurales calculadas.

Tabla 2. Muestra total de UPM y hogares POR zONAS, SEGÚN REgiones

\begin{tabular}{lccccc}
\hline \multicolumn{5}{c}{ Cálculo de la muestra total de UPM y hogares según regiones } \\
\hline \multicolumn{1}{c}{ Regiones } & Hogares & RQ-H & UPM & n & e.m. \\
\hline Cibao Norte & 502,170 & 708.64 & 162 & 808 & 2.1 \\
Cibao Sur & 233,653 & 483.38 & 110 & 551 & 2.6 \\
Cibao Nordeste & 221,006 & 470.11 & 107 & 536 & 2.6 \\
Cibao Noroeste & 137,840 & 371.27 & 85 & 423 & 3.0 \\
Valdesia & 310,369 & 557.11 & 127 & 635 & 2.4 \\
Enriquillo & 104,039 & 322.55 & 74 & 368 & 3.2 \\
El Valle & 99,148 & 314.88 & 72 & 359 & 3.2 \\
Yuma & 177,855 & 421.73 & 96 & 481 & 2.8 \\
Higuamo & 198,235 & 445.24 & 102 & 508 & 2.7 \\
Ozama & 911,488 & 954.72 & 218 & 1,089 & 1.8 \\
Total & $2,895,803$ & 5049.62 & 1152 & 5,758 & 0.8
\end{tabular}




\begin{tabular}{lccccc}
\hline \multicolumn{5}{c}{ Distribución muestra UPM y hogares por zonas según regiones } \\
\hline \multicolumn{1}{c}{ Regiones } & UPM urbanas & $\begin{array}{c}\text { Hogares ur- } \\
\text { banos }\end{array}$ & $\begin{array}{c}\text { UPM } \\
\text { rurales }\end{array}$ & $\begin{array}{c}\text { Hogares } \\
\text { rurales }\end{array}$ & $\begin{array}{c}\text { Muestra } \\
\text { total }\end{array}$ \\
\hline Cibao Norte & 89 & 445 & 73 & 363 & 808 \\
Cibao Sur & 55 & 273 & 56 & 278 & 551 \\
Cibao Nordeste & 52 & 259 & 56 & 278 & 537 \\
Cibao Noroeste & 45 & 225 & 40 & 199 & 424 \\
Valdesia & 65 & 325 & 62 & 310 & 635 \\
Enriquillo & 43 & 217 & 30 & 151 & 368 \\
El Valle & 34 & 170 & 38 & 189 & 359 \\
Yuma & 58 & 291 & 38 & 190 & 481 \\
Higuamo & 59 & 295 & 43 & 213 & 508 \\
Ozama & 166 & 828 & 52 & 261 & 1,089 \\
Total & 665 & 3,327 & 486 & 2,430 & 5,757 \\
\hline
\end{tabular}

Notas. RQ-H: raíz cuadrada de los hogares; e.m.: error muestral.

Fuente. Elaborado con datos de la ENFT 2013 del Banco Central de la República Dominicana.

La tabla 3 presenta la muestra efectiva levantada, la cual totaliza unas 5,803 entrevistas, sobrepasando con 46 entrevistas la muestra planificada.

Tabla 3. Muestra de hogares levantados por zonas urbana, Rural y Regiones

\begin{tabular}{lccc}
\hline \multirow{2}{*}{ Regiones } & \multicolumn{3}{c}{ Zona } \\
\cline { 2 - 4 } & Rural & urbana & Total \\
\hline Ozama & 98 & 995 & 1093 \\
Cibao Norte & 361 & 449 & 810 \\
Cibao Sur & 270 & 280 & 550 \\
Cibao Nordeste & 245 & 290 & 535 \\
Cibao Noroeste & 200 & 230 & 430 \\
Yuma & 154 & 341 & 495 \\
Higuamo & 209 & 283 & 492 \\
Valdesia & 375 & 300 & 675 \\
El Valle & 198 & 167 & 365 \\
Enriquillo & 145 & 221 & 366 \\
Total & 2,415 & 3,395 & 5,810 \\
\hline
\end{tabular}

Fuente. Elaborado con datos de la ENFT 2013 del Banco Central de la República Dominicana. 


\section{E. Factores de ponderación y expansión de la muestra}

Por definición, el factor de ponderación de la muestra es igual al inverso de la probabilidad de selección de las unidades muestrales.

El muestreo aplicado fue bietápico, por tanto, la probabilidad de selección de un hogar es el producto de la probabilidad de selección de una UPM (primera etapa), multiplicado por la probabilidad de selección de un hogar en la segunda etapa (selección dentro de la UPM). El procedimiento se expresa de la siguiente manera.

$$
\frac{1}{p_{i}}=F p
$$

Donde:

Probabilidad de selección primera etapa: $\quad \frac{m_{h i}}{\mathrm{M}} * \mathrm{~A}_{h} * b_{h}=p_{1}$

Probabilidad de selección segunda etapa: $\quad \frac{n_{h i}}{M_{h i}}=p 2$

$\mathrm{m}_{\text {hi }}$ : número de hogares dentro de cada UPM del estrato $\mathrm{h}$

M: total de hogares de un estrato

$\mathrm{A}_{\mathrm{h}}$ : número de UPM de un estrato

$\mathrm{b}_{\mathrm{h}}$ : cociente del número de hogares seleccionados y el total de hogares de una UPM en el estrato $h$.

$\mathrm{n}_{\text {hi: }}$ número de hogares seleccionados en cada UPM del estrato $\mathrm{h}$.

$\mathrm{n}_{\mathrm{he}}$ : número de hogares entrevistados en cada UPM del estrato $\mathrm{h}$

Para la presente encuesta, el factor de ponderación de diseño se calculó de la siguiente manera;

$$
\frac{p_{1}}{p_{2}}=F 1
$$


El factor de ponderación de diseño (F1) fue ajustado por sexo y zona de residencia a nivel de las diez regiones del país, utilizando la Encuesta Nacional de Fuerza de trabajo. El ponderador final se obtuvo de la siguiente forma:

$$
F=F_{1} * F_{\text {sex }} * F_{\text {zona }}
$$

Luego, los factores de expansión se obtuvieron de la siguiente manera:

$$
\frac{N_{h}}{n_{h e}} * F_{h}=F_{\text {exp }}
$$

En la que:

Nh: es el número de hogares estimados a partir de las proyecciones de población de la Oficina Nacional de Estadística para el año 2015 para cada una de las regiones. Luego, el total país se obtuvo por agregación de las 10 regiones de planificación.

\section{Selección de la muestra}

Las unidades primarias de muestreo son las UPM y las unidades secundarias son los hogares en cada UPM, con un tamaño fijo de cinco (5) viviendas por cada UPM, y los informantes fueron los jefes o las jefas de hogares. Se aplicó un proceso aleatorio de selección, tanto en la primera etapa (selección de UPM) como en la segunda (selección de hogares). Cuando salió la muestra de hogares (segunda etapa), viviendas con 2,3...Xn hogares, se procedió a seleccionar uno de forma aleatoria.

En resumen, la muestra diseñada y aplicada es probabilística, bietápica y estratificada, con selección aleatoria, y de forma independiente en cada dominio, ponderada y expandida. 
Todos los cruces de variables realizados y mostrados en las tablas del informe fueron evaluados en análisis de varianza (ANOVA) ${ }^{2}$ y resultaron significativos en pruebas de Pearson $^{3}$, así como en otras medidas de asociación entre las variables.

\section{Principales características de los hogares}

Tabla 4. Ubicación de los hogares encuestados por Regiones de DeSARROLlo

\begin{tabular}{lcccc}
\hline Regiones & Rural & Urbana & Total & Porcentaje \\
\hline Ozama & 98 & 995 & 1093 & 18.81 \\
Cibao Norte & 324 & 486 & 810 & 13.94 \\
Valdesia & 318 & 357 & 675 & 11.58 \\
Cibao Sur & 279 & 271 & 550 & 9.46 \\
Cibao Nordeste & 289 & 246 & 535 & 9.21 \\
Yuma & 148 & 348 & 497 & 8.55 \\
Higuamo & 168 & 325 & 493 & 8.50 \\
Cibao Noroeste & 232 & 200 & 432 & 7.44 \\
Enriquillo & 119 & 247 & 366 & 6.30 \\
El Valle & 197 & 168 & 365 & 6.21 \\
Nacional & 2,171 & 3,639 & 5,810 & 100.00 \\
\hline
\end{tabular}

Fuente. Encuesta de suministro eléctrico a hogares (ESEH), INTEC-BID (2015).

En el levantamiento realizado durante los meses de enero y febrero de 2015, la mayoría de los hogares encuestados pertenece a la Región Ozama (18.81\%). De estos, el 91\% está ubicado en la zona urbana, mientras que solo el $9 \%$ se encuentra en la zona rural.

La región Cibao Norte es la segunda con mayor porcentaje de hogares encuestados (13.94\%). En esta región, el 60\% de los hogares

\footnotetext{
${ }^{2}$ El análisis de la varianza (ANOVA) es un método para comparar dos o más medias, que es necesario porque cuando se quiere hacer dicha comparación es incorrecto utilizar repetidamente el contraste basado en la t de Student.

${ }^{3}$ La prueba $\chi^{2}$ de Pearson es una prueba no paramétrica que mide la discrepancia entre una distribución observada y otra teórica (bondad de ajuste), indicando en qué medida las diferencias existentes entre ambas se deben al azar en el contraste de hipótesis. También se utiliza para probar la independencia de dos variables entre sí, mediante la presentación de los datos en tablas de contingencia.
} 
está ubicado en la zona urbana y el resto en la zona rural. En Valdesia, se encuentra el $11.58 \%$ de los hogares que componen la muestra. En esta región, la mayoría de hogares se encuentra en la zona rural (53.04\%). Las regiones Cibao Sur, Nordeste y Noroeste representan el $9.46 \%, 9.21 \%$ y $7.44 \%$ de los hogares encuestados. En estas regiones la mayoría de los hogares está ubicada en la zona rural con $50.9 \%$, $53.9 \%$ y $53.6 \%$, respectivamente (ver tabla 4 ).

Las regiones Enriquillo y El Valle representan el 6.30\% y 6.21\% de la muestra seleccionada. De estas, solo en la región del Valle la mayoría de los hogares está ubicada en la zona rural (54.6\%).

Tabla 5. Ubicación de los hogares encuestados por Regiones de Desarrollo

\begin{tabular}{lcccc}
\hline Regiones & Hombre & Mujer & Total & Porcentaje \\
\hline Ozama & 675 & 417 & 1092 & 18.81 \\
Cibao Norte & 513 & 297 & 810 & 13.94 \\
Valdesia & 477 & 196 & 673 & 11.58 \\
Cibao Sur & 363 & 187 & 550 & 9.46 \\
Cibao Nordeste & 368 & 167 & 535 & 9.21 \\
Yuma & 326 & 171 & 497 & 8.55 \\
Higuamo & 324 & 169 & 493 & 8.50 \\
Cibao Noroeste & 293 & 139 & 432 & 7.44 \\
Enriquillo & 267 & 99 & 366 & 6.30 \\
El Valle & 265 & 100 & 365 & 6.21 \\
Nacional & 3,868 & 1,942 & 5,810 & 100.00 \\
\hline
\end{tabular}

Fuente. Encuesta de suministro eléctrico a hogares (ESEH), INTEC-BID (2015).

Con relación al género de jefe del hogar, el $64.57 \%$ del total de encuestados son hombres y el $35.43 \%$ son mujeres.

Sin embargo, estos porcentajes varían de acuerdo con la región de desarrollo. En la región Ozama, el porcentaje de hogares cuyo jefe de hogar es un hombre es menor al promedio (62\%), así como en la región Cibao Norte (63\%). En las regiones Cibao Sur, Nordeste y Noroeste los porcentajes están por encima del promedio con $66 \%, 69 \%$ y $68 \%$, respectivamente. 
En las regiones de Enriquilo y El Valle los porcentajes de hogares cuyo jefe de hogar es un hombre se elevan a $72.9 \%$ y $72.3 \%$, respectivamente (ver tabla 5).

En cuanto a los hogares cuya jefa de hogar es una mujer, existe una relación inversa entre el porcentaje de hogares ubicados en la zona rural y la jefatura femenina del hogar. A mayor porcentaje de hogares en la zona rural, menor porcentaje de mujeres se desempeña como jefas de hogar.

En lo referente a la edad del jefe de hogar, el 32.3\% tiene 55 años o más; el $28.4 \%$ tiene entre 35 y 54 años; un $21.4 \%$ entre 35 y 44 años. Los grupos etarios de 25-34 años y de 18-24 años tienen menores porcentajes con $12.6 \%$ y $5.3 \%$, respectivamente (ver tabla 6 ).

Los porcentajes varían de acuerdo a las regiones de desarrollo evaluadas. En la región El Valle es donde existe un mayor porcentaje de jefes de hogar mayores de 55 años (45.3\%), seguido por Ozama (37.9\%) y Cibao Nordeste (37.0\%). En Cibao Norte es donde hay un menor porcentaje de jefes de hogar mayores de 55 años (21.3\%).

Tabla 6. Rango DE EDAD, EN AÑos, DEl JEFE DE hOGAR POR REgIONES DE DESARRollo

\begin{tabular}{lccccccc}
\hline Regiones & $18-24$ & $25-34$ & $35-44$ & $45-54$ & $\begin{array}{c}55 \text { ó } \\
\text { mas }\end{array}$ & Total & $\begin{array}{c}\text { Porcen- } \\
\text { taje }\end{array}$ \\
\hline Ozama & 53 & 178 & 206 & 241 & 415 & 1,093 & 18.81 \\
Cibao Norte & 15 & 83 & 251 & 288 & 171 & 810 & 13.94 \\
Valdesia & 37 & 96 & 135 & 168 & 238 & 674 & 11.58 \\
Cibao Sur & 18 & 44 & 154 & 191 & 142 & 549 & 9.46 \\
Cibao Nordeste & 19 & 65 & 107 & 146 & 198 & 535 & 9.21 \\
Yuma & 17 & 47 & 88 & 228 & 116 & 496 & 8.55 \\
Higuamo & 67 & 75 & 96 & 91 & 166 & 495 & 8.50 \\
Cibao Noroeste & 28 & 48 & 102 & 118 & 135 & 431 & 7.44 \\
Enriquillo & 32 & 52 & 54 & 96 & 133 & 367 & 6.30 \\
El Valle & 22 & 43 & 50 & 82 & 163 & 360 & 6.21 \\
Nacional & 308 & 731 & 1,243 & 1,649 & 1,879 & 5,810 & 100.00 \\
\hline
\end{tabular}

Fuente. Encuesta de suministro eléctrico a hogares (ESEH), INTEC-BID (2015). 
En términos generales, el $67.7 \%$ de los jefes de hogar son menores de 54 años. En la región Ozama y Cibao Nordeste este porcentaje es de $62.1 \%$ y 63\%, respectivamente. En Cibao Norte y Cibao Sur esta cifra se incrementa, alcanzando un $78.8 \%$ en la primera y un $74.1 \%$ en la segunda.

Con relación al nivel educativo del jefe del hogar, los resultados fueron los siguientes: el 7.3\% no tiene ningún grado académico; un $46.59 \%$ tiene nivel primario; el $31.53 \%$ tiene un nivel secundario o técnico; el $12.72 \%$ es universitario; y un $1.9 \%$ tiene grado post-universitario (ver tabla 7).

El nivel educativo del jefe de hogar varía según la región de desarrollo donde habita. En las regiones Ozama, Cibao Norte y Cibao Nordeste existe un mayor porcentaje de jefes de hogar con estudios universitarios, con $21.3 \%, 13.8 \%$ y $8.7 \%$, respectivamente.

Tabla 7. Nivel educativo del JeFe De hogar POR REgiones DE DESARRollo

\begin{tabular}{lccccccc}
\hline Regiones & $\begin{array}{c}\text { Ningu- } \\
\text { no }\end{array}$ & $\begin{array}{c}\text { Prima- } \\
\text { rio }\end{array}$ & $\begin{array}{c}\text { Secun- } \\
\text { dario } \\
\text { técnico }\end{array}$ & $\begin{array}{c}\text { Univer- } \\
\text { sitario }\end{array}$ & $\begin{array}{c}\text { Post } \\
\text { univer- } \\
\text { sitario }\end{array}$ & Total & $\begin{array}{c}\text { Porcen- } \\
\text { taje }\end{array}$ \\
\hline Ozama & 54 & 426 & 359 & 205 & 49 & 1,093 & 18.81 \\
Cib. Norte & 5 & 283 & 409 & 102 & 11 & 810 & 13.94 \\
Valdesia & 55 & 336 & 214 & 62 & 7 & 674 & 11.58 \\
Cibao Sur & 38 & 271 & 181 & 54 & 5 & 549 & 9.46 \\
Cib. Nordeste & 47 & 238 & 177 & 64 & 9 & 535 & 9.21 \\
Yuma & 35 & 296 & 98 & 60 & 7 & 496 & 8.55 \\
Higuamo & 32 & 256 & 158 & 43 & 6 & 495 & 8.50 \\
Cib. Noroeste & 55 & 224 & 103 & 40 & 9 & 431 & 7.44 \\
Enriquillo & 43 & 201 & 75 & 47 & 1 & 367 & 6.30 \\
El Valle & 58 & 176 & 58 & 62 & 6 & 360 & 6.21 \\
Nacional & 422 & 2,707 & 1,832 & 739 & 110 & 5,810 & 100.00 \\
\hline
\end{tabular}

Fuente. Encuesta de suministro eléctrico a hogares (ESEH), INTEC-BID (2015).

La composición de los miembros del hogar por grupos etarios es otro de los elementos relevantes a la hora de establecer el consumo 
de energía de las viviendas. En los hogares de las regiones Valdesia y Enriquillo hay un mayor porcentaje de miembros mayores de 18 años, con 2.75 y 2.74 personas por hogar, respectivamente.

En las regiones Cibao Sur e Higuamo es donde hay un mayor porcentaje de niños menores de 6 años por hogar, con $0.46 \%$ en la primera y $0.45 \%$ en la segunda (ver tabla 8 ).

TABla 8. Composición de miembros del hogar POR REgiones de DESARRollo

\begin{tabular}{lccccc}
\hline Regiones & $\begin{array}{c}\text { Número } \\
\text { hogares }\end{array}$ & $\begin{array}{c}\text { Mayores } \\
18 \text { años }\end{array}$ & $\begin{array}{c}12-17 \\
\text { años }\end{array}$ & 6-11 años & $\begin{array}{c}\text { Menores } \\
6 \text { años }\end{array}$ \\
\hline Ozama & 1,093 & 2.59 & 0.35 & 0.29 & 0.44 \\
Cibao Norte & 810 & 2.67 & 0.36 & 0.37 & 0.38 \\
Valdesia & 673 & 2.75 & 0.51 & 0.36 & 0.39 \\
Cibao Sur & 550 & 2.66 & 0.35 & 0.31 & 0.46 \\
Cibao Nordeste & 535 & 2.54 & 0.39 & 0.36 & 0.38 \\
Yuma & 496 & 2.63 & 0.31 & 0.29 & 0.27 \\
Higuamo & 493 & 2.44 & 0.39 & 0.42 & 0.45 \\
Cibao Noroeste & 432 & 2.43 & 0.37 & 0.31 & 0.37 \\
El Valle & 361 & 2.69 & 0.43 & 0.41 & 0.41 \\
Enriquillo & 366 & 2.74 & 0.53 & 0.43 & 0.60 \\
\hline
\end{tabular}

Notas. Valores medios para todos los rangos de edades mostrados.

Fuente. Encuesta de suministro eléctrico a hogares (ESEH), INTEC-BID (2015).

En las regiones Cibao Nordeste e Higuamo hay un mayor porcentaje de jóvenes entre 12-17 años, ambas con un 0.39\%.

Por otro lado, se preguntó a los jefes de los hogares encuestados si recibían subsidio Bonoluz, otorgado a las familias de bajos ingresos que consumen menos de 200 kilovatios al mes y que están inscritos como beneficiarios en la Administradora de Subsidios Sociales (ADESS).

El 74.59\% de los jefes de hogar encuestados no recibe Bonoluz por la energía eléctrica que consume su vivienda mensualmente, mientras que el $25.41 \%$ sí lo recibe (ver tabla 9). 
Tabla 9. Hogares que Reciben/No ReCiben subsidio de Bonoluz por Regiones de DESARROLLO

\begin{tabular}{lccc}
\hline Regiones & No Recibe & Sí Recibe & Total \\
\hline Ozama & 889 & 204 & 1093 \\
Cibao Norte & 690 & 120 & 810 \\
Valdesia & 579 & 94 & 673 \\
Cibao Sur & 386 & 164 & 550 \\
Cibao Nordeste & 368 & 167 & 535 \\
Yuma & 411 & 85 & 496 \\
Higuamo & 306 & 188 & 494 \\
Cibao Noroeste & 284 & 148 & 432 \\
El Valle & 177 & 183 & 360 \\
Enriquillo & 242 & 124 & 366 \\
Porc. (\%) & 74.59 & 25.41 & 100 \\
\hline
\end{tabular}

Fuente. Encuesta de suministro eléctrico a hogares (ESEH), INTEC-BID (2015).

En la región El Valle, el 50.83\% de los hogares recibe Bonoluz, siendo la mayor de todas las regiones encuestadas. En las regiones de Higuamo, Cibao Noroeste y Enriquillo los hogares que reciben Bonoluz represen$\tan$ el $38.06 \%, 34.11 \%$ y $33.88 \%$, respectivamente. En Valdesia es donde existe un menor porcentaje de hogares que recibe Bonoluz (13.97\%), por debajo de las regiones Ozama (18.66\%) y Cibao Norte (14.81\%).

Por otro lado, el gasto promedio mensual de todos los hogares evaluados a nivel nacional es de $\mathrm{RD} \$ 15,837.85$. Los hogares de la región Ozama se encuentran por encima de la media con $\mathrm{RD} \$ 20,126.0$ de gasto mensual, seguido por los de Cibao Norte con $\mathrm{RD} \$ 16,536.75$ (ver tabla 10).

El gasto mensual promedio de los hogares de las regiones Yuma y Cibao Sur es de $\mathrm{RD} \$ 16,186.87$ y $\mathrm{RD} \$ 15,884.93$, respectivamente. Los hogares de la región Enriquillo son los que tienen menor gasto mensual con solo RD $\$ 12,017.09$. 
Tabla 10. Gasto total mensual del hogar por Regiones de Desarrollo

\begin{tabular}{lccccccc}
\hline Regiones & Hogares & Media & Desv.Est. & Mediana & Moda & Mínimo & Máximo \\
\hline Ozama & 1,073 & $20,126.0$ & $15,667.4$ & $16,000.00$ & 15,000 & 1,000 & 250,000 \\
Cibao Norte & 760 & $16,536.75$ & $6,442.9$ & $16,000.00$ & 15,000 & 3,000 & 70,000 \\
Valdesia & 666 & $14,898.11$ & $8,883.3$ & $13,000.00$ & 15,000 & 2,500 & 70,000 \\
Cibao Sur & 550 & $15,884.93$ & $8,447.91$ & $15,000.00$ & 15,000 & 3,500 & 150,000 \\
Cibao Nor- & 535 & $15,839.48$ & $9,576.5$ & $15,000.00$ & 15,000 & 3,000 & 100,000 \\
deste & 486 & $16,186.87$ & $8,450.6$ & $15,000.00$ & 15,000 & 3,000 & 45,000 \\
Yuma & 485 & $13,129.38$ & $8,610.3$ & $12,000.00$ & 10,000 & 2,500 & 120,000 \\
Higuamo & 432 & $13,111.26$ & 9.157 .1 & $12,000.00$ & 10,000 & 2,500 & 150,000 \\
Cibao No- & & $13,579.12$ & $8,178.3$ & $12,000.00$ & 15,000 & 2,500 & 80,000 \\
roeste & 360 & $12,017.09$ & $7,546.2$ & $10,000.00$ & 15,000 & 2,500 & 50,000 \\
El Valle & 366 & $15,837.85$ & $10,437.9$ & $15,000.00$ & 15,000 & 1,000 & 250,000 \\
Enriquillo & 5,698 & 15,000 \\
Nacional & & & & & &
\end{tabular}

Fuente. Encuesta de suministro eléctrico a hogares (ESEH), INTEC-BID (2015).

\section{Disponibilidad de computador, acceso a Internet y otros servicios de telecomunicaciones}

Una de las premisas en la discusión del cuestionario a aplicar para la ESEH 2015 era la posibilidad de establecer un módulo de telecomunicaciones y definir los niveles de disponibilidad y acceso a los distintos servicios finales de telecomunicaciones, especialmente al Internet.

Al preguntar sobre la disponibilidad o no de algún equipo de computación en el hogar que tuviera la capacidad de conectarse al Internet, el $28.11 \%$ del total de encuestados respondió que tenía al menos un desktop, laptop o tablet en la vivienda.

En la región Ozama es donde existe un mayor porcentaje de hogares con computador $(35.0 \%)$, seguido por las regiones de Cibao Norte y Valdesia con $34.12 \%$ y $32.04 \%$, respectivamente (ver tabla 11). 
Sin embargo, tener un computador es una condición necesaria, pero no suficiente para contratar el servicio de Internet en el hogar, debido a que existen otros factores que intervienen en la decisión vinculados a la disponibilidad o no del servicio en el área de la vivienda, así como al costo del mismo.

TABla 11. DisponibilidAD DE COMPUTADOR (DESKTOP, LAPTOP O TABLET) EN EL HOGAR POR REGIONES DE DESARROLLO

\begin{tabular}{lccc}
\hline Regiones & No Posee & Sí Posee & Total \\
\hline Ozama & 826 & 383 & 1093 \\
Cibao Norte & 568 & 284 & 810 \\
Valdesia & 588 & 216 & 674 \\
Cibao Sur & 445 & 165 & 550 \\
Cibao Nordeste & 470 & 161 & 535 \\
Yuma & 435 & 99 & 495 \\
Higuamo & 452 & 98 & 492 \\
Cibao Noroeste & 356 & 108 & 430 \\
El Valle & 327 & 54 & 360 \\
Enriquillo & 330 & 66 & 366 \\
Porc. (\%) & 71.89 & 28.11 & 100 \\
\hline
\end{tabular}

Fuente. Encuesta de suministro eléctrico a hogares (ESEH), INTEC-BID (2015).

A la pregunta de si tenían o no servicio de televisión por cable en el hogar, el 61.5\% respondió que no dispone de ese servicio. En las regiones Enriquillo e Higuamo es donde hay un mayor porcentaje de hogares que carecen de este servicio $(74.9 \%$ y $74.5 \%)$. En Valdesia y El Valle los porcentajes son menores, con $73.1 \%$ y $66.5 \%$ (ver figura 1). 
Figura 1. Hogares Con televisión POR CABLE POR Regiones de DeSARrollo

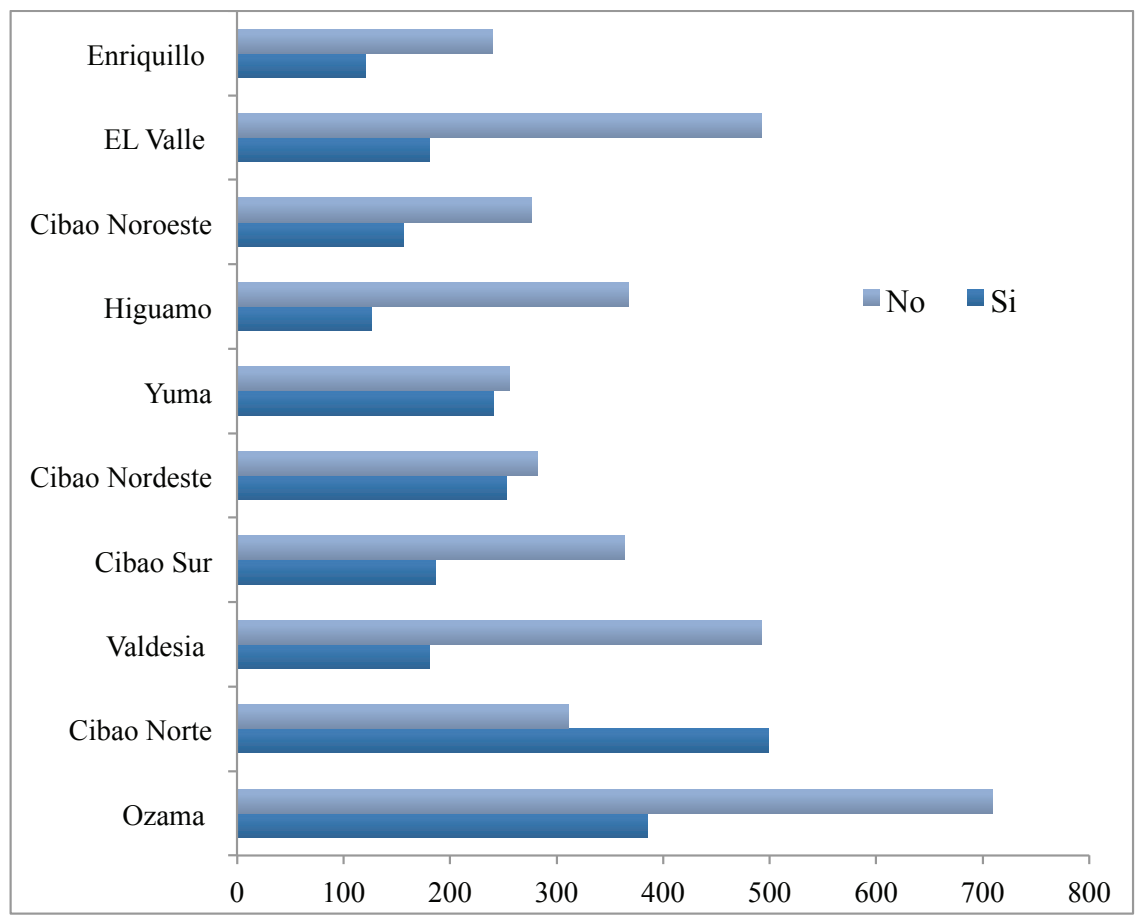

Fuente: encuesta de suministro eléctrico a hogares (ESEH), INTEC-BID (2015).

Cibao Norte es la única región donde la cantidad de hogares que tiene telecable supera a los que no tienen (61.6\% vs. 38.4\%). La tarifa promedio que pagan los hogares oscilaba entre RD $\$ 1,042.22$, en Ozama, y RD\$580.39, en Cibao Noroeste.

Más adelante, se preguntó a los encuestados si tenían teléfono residencial y si junto a este tenían acceso a Internet en el hogar. De acuerdo con los resultados, solo el $35.79 \%$ del total de hogares encuestados tiene teléfono residencial. De estos, los hogares de las regiones Ozama y Cibao Norte tienen los mayores porcentajes, con $49.3 \%$ y $44.3 \%$, respectivamente. 
El porcentaje de hogares de Yuma y Cibao Sur que tiene telefonía residencial es de $30.9 \%$, la primera, y $24.5 \%$, la segunda. Los hogares establecidos en El Valle tienen el menor porcentaje, con un 14.1\% (ver figura 2).

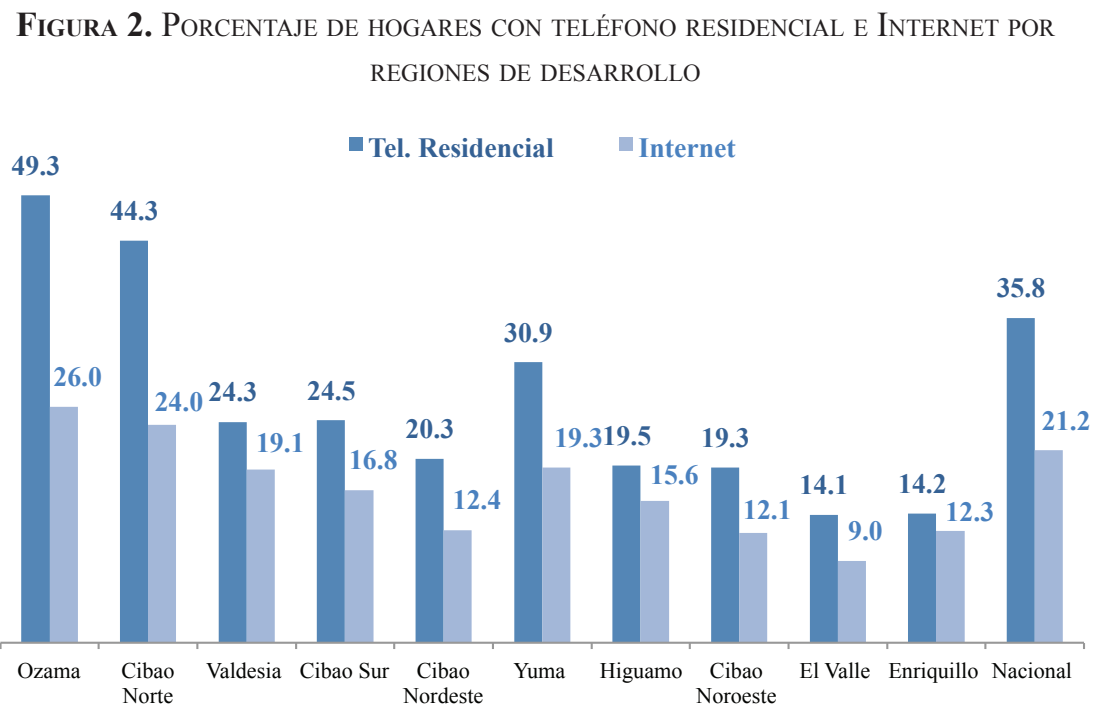

Fuente: encuesta de suministro eléctrico a hogares (ESEH), INTEC-BID (2015).

Otro hallazgo que llama la atención es la baja penetración de Internet en los hogares. Del total de hogares encuestados, solo el 16.1\% tiene Internet. El porcentaje de hogares de las regiones Ozama y Cibao Norte es mayor al promedio, con $26.0 \%$ y $22.0 \%$, respectivamente.

Los hogares de Yuma y Valdesia alcanzan porcentajes menores $(17.3 \%$ y $13.1 \%)$. Cibao Nordeste es la región donde menor porcentaje de hogares tiene acceso a internet (8.4\%).

Las tarifas promedio que pagan los hogares por el servicio de telefonía fija e Internet oscila entre RD\$1,325.29, en Ozama, y RD \$858.19, en Cibao Noroeste.

Para completar la sección de telecomunicaciones preguntamos al jefe de hogar si tenía teléfono móvil. Según los resultados, el 80.5\% 
de los jefes de hogar encuestados tiene teléfono móvil. Curiosamente, el resultado es más alto en la Región El Valle (86.9\%).

Las regiones Cibao Norte y Cibao Nordeste tienen porcentajes de $86.0 \%$ y $81.7 \%$, respectivamente. En Higuamo y Yuma los porcentajes son menores al $77 \%{ }^{4}$ (ver figura 3 ).

Figura 3. Porcentaje de Jefes de hogar CON teléfono MÓvil POR REGiOnes de DESARROLLO

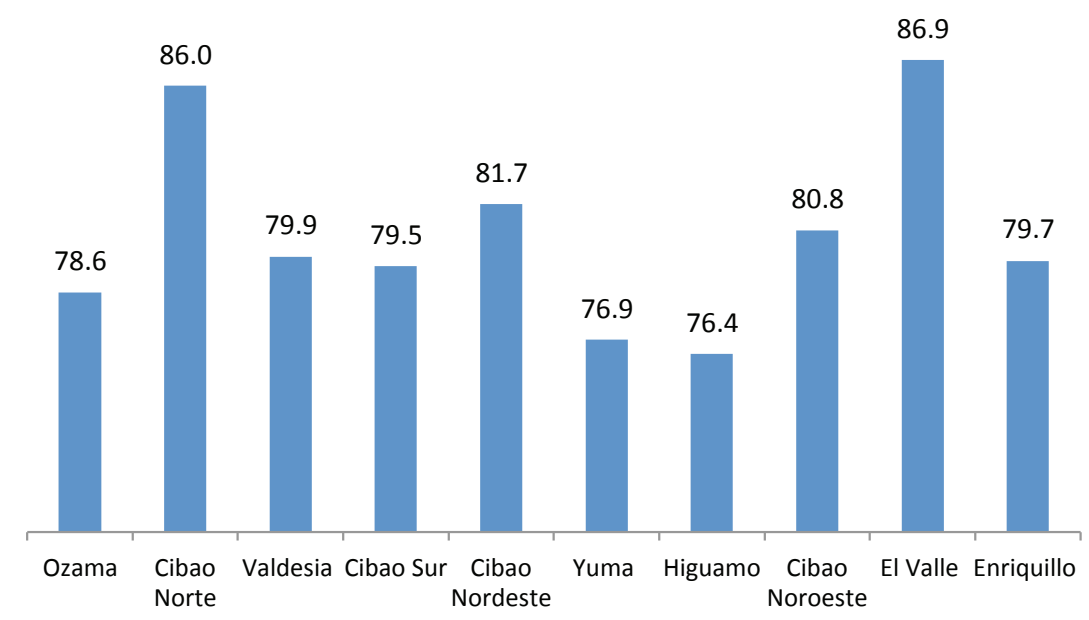

Fuente: encuesta de suministro eléctrico a hogares (ESEH), INTEC-BID (2015).

Los entrevistados señalaron que las tarifas que pagan mensualmente por concepto de móviles oscilan entre RD\$692, en Ozama, y RD\$418.35, en Cibao Noroeste.

\section{Marco teórico}

Existe una vasta literatura en torno al impacto del capital humano en la productividad, el crecimiento económico y el bienestar de las personas. Si se considera que el Internet es una fuente de conocimiento

\footnotetext{
${ }^{4}$ Este dato podría diferir de la penetración real en móviles debido a que en el hogar puede haber más de un abonado a este servicio. El hecho de haber entrevistado solo al jefe de hogar podría subestimar la cantidad de móviles de los que habitan en la vivienda.
} 
e información, este tendría la capacidad de fortalecer el capital humano de los usuarios del servicio de telecomunicaciones.

Por tal razón, es de esperarse que el uso de Internet genere diferenciales en términos de ingresos entre aquellos hogares que tienen acceso a este y aquellos que no.

Varios estudios se han centrado en la identificación de otras posibles determinantes de la penetración de computadoras e Internet. Por ejemplo, Goolsbee \& Klenow (2002) investigaron sobre los efectos de la concentración geográfica en la tenencia de computadores. Encontraron que en Estados Unidos es más probable que la gente posea computadoras en áreas donde hay una mayor concentración de personas.

Por otro lado, Greenstein \& Prince (2006) analizan la difusión geográfica del Internet en Estados Unidos para hogares y empresas. Bajo el marco de la teoría de la difusión económica, estos autores concluyen que el Internet primero se desarrolló temporalmente en varias zonas urbanas debido a sus recursos complementarios. Una vez que las aplicaciones maduraron, las áreas líderes perdieron su posición y los proveedores de servicios de Internet ampliaron la comercialización al ámbito internacional.

Venkatesh \& Shih (2005) investigaron cómo las diferentes teorías de la difusión - evolucionaria, estructural, de agencia, entre otras- sirven para explicar la difusión empírica de Estados Unidos, Suecia y la India con el fin de obtener un mejor entendimiento de la forma en que se integra el uso de la tecnología en los hogares ${ }^{5}$.

Estos autores encontraron que ninguna teoría en particular puede explicar de forma exclusiva todos los desarrollos y que las mismas se aplican con diferentes grados. Sostienen que los determinantes

\footnotetext{
${ }^{5}$ El modelo de aceptación de la tecnología pivota en torno a tres ejes fundamentales: la utilidad percibida de la tecnología (UP), la facilidad de uso de la tecnología (FUP) y las actitudes sobre la tecnología (AT) (Venkatesh et al., 2003).
} 
que explican por qué los computadores están integrados en los hogares son similares entre culturas, haciendo hincapié en el papel del impacto en el bienestar, dado el nivel de uso de la tecnología en el hogar. Los resultados también confirman la fuerte correlación entre el uso de computadoras y otras tecnologías de información y comunicación.

Uno de los estudios más citados sobre este tema corresponde a Gi-Soon \& Song (2005), que utilizando datos de una muestra panel de hogares rurales de la República Democrática de Lao, estima el cambio en los gastos del hogar, en función de la tenencia de servicios de Internet.

En este estudio, se elabora un modelo conceptual que desarrolla la cadena de transmisión entre el acceso y uso a servicios de Internet y las mejoras económicas y sociales en el hogar. El modelo plantea, en esencia, que el acceso y uso de Internet permite la toma de mejores decisiones (o decisiones más informadas), a través de la reducción en los costos de transacción y de la incertidumbre, derivados del acceso a más y mejor información, así como de los ahorros de costos y de tiempo para acceder a la misma.

Los resultados muestran que la presencia de este servicio tiene un impacto significativo en el bienestar de los hogares. Asimismo, evidencia que el uso del servicio aumenta el consumo, principalmente en los hogares con menores recursos.

De igual modo, Trung et al. (2007), utilizaron el mismo modelo para Vietnam, encontrando impactos significativos del uso de las tecnologías de la información y comunicación sobre el ingreso ${ }^{6}$.

\footnotetext{
${ }^{6}$ El estudio finalmente excluyó el uso de internet por su alta correlación con otras variables como el uso de telefonía móvil y la tenencia de computadoras.
} 
Vicente \& López (2006) encontraron evidencia de que el ingreso y la educación superior son determinantes, tanto para la adopción de computadoras como para el uso de Internet.

Otros estudios han desarrollado diversas técnicas para encontrar los determinantes del uso de Internet. Por ejemplo, Demousis \& Giannakopoulus (2006), apelando al modelo de probabilidades ordenado, han encontrado que para catorce países europeos el acceso a internet depende de variables como el ingreso, la educación, el género, la ubicación geográfica y el costo del servicio.

La ubicación geográfica del hogar es determinante para el uso de Internet, dadas las condiciones de accesibilidad al servicio y los costos de adquirirlo. Lo dicho anteriormente se evidencia con los estudios de Ono \& Zavodni (2007a), quienes estudian patrones del uso de computadores y acceso a Internet en EE. UU., Suecia, Japón, Korea y Singapur, encontrando que la división tecnológica es el reflejo de desigualdades económicas y sociales pre-existentes.

En la misma línea, Grazzi \& Vergara (2011), en un estudio para siete países de Latinoamérica, encuentran que tanto el ingreso como la educación, así como el área geográfica (urbano/rural) son determinantes para la difusión de las tecnologías de comunicación e información. Este estudio considera que la adopción de computadoras determina completamente la posibilidad del uso de Internet. Utilizando un modelo probabilístico bivariado de estimación simultánea, de tal manera que pueda medir los determinantes de las tecnologías de difusión e información por separado.

Un aspecto central al momento de evaluar los determinantes del gasto del hogar en telecomunicaciones y la posibilidad de acceso a uno o varios servicios finales es el empaquetamiento en la venta, provisión u oferta de los mismos. 
Continuando con el análisis regional, Peres \& Hilbert (2009) proporcionan información perspicaz sobre la difusión de las tecnologías de la información y comunicación (TIC) en América Latina. Entre otras cuestiones, se centran en comparar las diferentes dimensiones de la brecha digital de los países de la región respecto de los países desarrollados.

De acuerdo con los resultados, esta brecha estaba disminuyendo en torno a la telefonía móvil, pero está aumentando en términos de tenencia de computador en el hogar y acceso a Internet de banda ancha.

Gutiérrez \& Gamboa (2008) se centran en la brecha digital entre las personas de bajos ingresos en Colombia, México y Perú. Sus resultados muestran que la educación es el factor más importante y esto limita la reducción de la brecha con los países desarrollados.

\section{EI modelo}

Tomando como referencia los estudios de Fairlie (2004), Vicente \& López (2006) y Grazzi \&Vergara (2011), utilizamos una extensión del modelo aleatorio de utilidad lineal para estimar la decisión del hogar de tener o no una computadora.

La utilidad asociada con cada una de las dos situaciones supone una relación lineal en función de un conjunto de características socioeconómicas del hogar (Xi) y de un conjunto estocástico que representa errores no observables y de medición (عi).

Por lo tanto, la utilidad del hogar i asociada a tener una computadora $(\mathrm{Ui}, \mathrm{H})$ y no tenerla $(\mathrm{Ui}, \mathrm{N})$ puede expresarse como:

$$
\begin{aligned}
& U i, H=X i \beta H+\varepsilon i, H \\
& U i, N=X i \beta N+\varepsilon i, N
\end{aligned}
$$


En tal sentido, el hogar i va a elegir tener un computador si la utilidad asociada con esta decisión es mayor que la utilidad asociada con no tener este equipo: $\mathrm{Ui}, \mathrm{H}>\mathrm{Ui}, \mathrm{N}$.

Si definimos una variable $\mathrm{Y}$ para que $\mathrm{Yi}, \mathrm{H}=1$ si el i-ésimo hogar posee un computador y $\mathrm{Yi}=0$ si no lo tiene. La probabilidad de que el i-ésimo hogar tenga acceso a un computador es $\operatorname{Pr}(\mathrm{Yi}, \mathrm{H}=1)=\operatorname{Pr}$ $(\mathrm{Ui}, \mathrm{H}>\mathrm{Ui}, \mathrm{N})=\Phi[\mathrm{Xi}(\beta \mathrm{H}-\beta \mathrm{N})]$, donde $\Phi$ es la función de distribu-

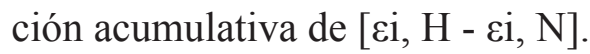

Al igualar la utilidad de no tener computador en el hogar a cero $(\mathrm{Ui}, \mathrm{N}=0$ ), derivamos la ecuación empírica para la adopción de la computadora:

$$
\operatorname{Pr}(\mathrm{Yi}, \mathrm{H}=1)=\operatorname{Pr}(\mathrm{Ui}, \mathrm{H}>0)=\Phi[\mathrm{Xi} \beta]
$$

De forma similar, podemos derivar una ecuación para modelar la probabilidad de que el hogar $\mathrm{j}$ tenga disponibilidad de Internet residencial:

$$
\operatorname{Pr}(Y j, C=1)=\operatorname{Pr}(U j, C>0)=\Phi[X j \theta]
$$

Donde $\mathrm{Uj}, \mathrm{C}$ es la utilidad indirecta asociada con la disponibilidad de Internet residencial.

Por lo tanto, podemos analizar empíricamente los determinantes de que el hogar disponga de un computador e Internet a través de la estimación de los parámetros $\beta$ y $\theta$ en las ecuaciones empíricas (7) y (8).

Un enfoque econométrico común para estimar estas ecuaciones por máxima verosimilitud es a través de un modelo Probit.

El modelo Probit asume que el término de error se distribuye normalmente con la media 0 y varianza $(\sigma)$ igual a 1 , así como $\Phi($.$) corres-$ ponden a la función de distribución normal acumulativa estandarizada. 
Sin embargo, un potencial problema con este enfoque es que no considera la correlación entre las elecciones de los hogares respecto del computador y el Internet. Por un lado, la adquisición de computadoras es un requisito previo para tener conexión a Internet disponible en casa.

Por otro lado, la decisión de adquirir un computador se puede fundar en el hecho de que es un paso previo necesario para poder disponer del Internet residencial. El punto clave es que un computador puede ser un bien final o simplemente un requisito para acceder a la web, dependiendo de las características del uso de la computadora en el hogar.

El vínculo entre estas decisiones plantea una importante consideración econométrica. De hecho, la estimación por máxima verosimilitud de dos modelos Probit correlacionados parece no ser una elección de procedimiento econométrico eficiente, ya que ignora la correlación entre los términos de error.

Dada la relación existente entre la disponibilidad de computador en el hogar y la posibilidad de acceder al Internet residencial, podría entenderse que lo más adecuado para estimar la significancia estadística de los parámetros sería utilizar un modelo de ecuaciones simultáneas.

Utilizando la función de la media condicional apropiada, se podría estimar consistentemente los parámetros de modelos de elección binaria utilizando mínimos cuadrados no lineales, pero estos también serían ineficientes y es preferible estimarlos por máxima verosimilitud.

En otro sentido, un modelo de regresión en dos etapas proporcionaría estimadores consistentes y errores estándar asintóticos apropiados, pero este enfoque tiene varias deficiencias. En primer lugar, ninguna especificación del lado derecho de la ecuación es una función de 
media condicional. Y, en segundo lugar, sería potencialmente ineficiente en la medida en que no tiene en cuenta la posible correlación entre las perturbaciones implícitas en las dos ecuaciones.

El procedimiento en dos pasos fue propuesto por Rivers y Vuong (1987) para modelos Probit en el que una o más de las variables del lado derecho son endógenas, pero requiere que sean continuas. Así, el uso del residuo en la segunda ecuación sería apropiado si fuera una variable continua, pero no para un modelo binario.

Esta técnica establece la estimación de probabilidad máxima condicional en el segundo paso, pero no en los mínimos cuadrados no lineales. No obstante, existe un estimador coherente y totalmente eficiente para este tipo de modelo que es en realidad mucho más sencillo de aplicar que el estimador de dos pasos, basado en las relaciones entre las probabilidades conjuntas, condicionales y marginales.

El estimador, que proviene de una extensión del modelo Probit, toma en cuenta la correlación entre los términos de error y es conocido como Probit Bivariado o Biprobit (Greene, 2006).

En el modelo Probit Bivariado, los términos de error siguen una distribución normal bivariada:

$$
\begin{aligned}
& \operatorname{Pr}(\mathrm{Yi}, \mathrm{H}=1)=\Phi[\mathrm{Xi} \beta] \\
& \operatorname{Pr}(\mathrm{Yj}, \mathrm{C}=1)=\Phi[\mathrm{Xj} \theta] \\
& \mathrm{E}(\varepsilon \mathrm{i}, \mathrm{H})=\mathrm{E}(\varepsilon \mathrm{j}, \mathrm{C})=0 ; \mathrm{V}(\varepsilon \mathrm{i}, \mathrm{H})=\mathrm{V}(\varepsilon \mathrm{j}, \mathrm{C})=1 ; \operatorname{Cov}(\varepsilon \mathrm{i}, \mathrm{H}, \varepsilon \mathrm{j}, \\
& \mathrm{C})=\rho(11)
\end{aligned}
$$

El modelo se estima a través del método de máxima verosimilitud extendido, conocido como Máxima Verosimilitud con Información Completa (MVIC), dado que considera de forma simultánea la información contenida en todas las ecuaciones. 
La bondad de ajuste del modelo se mide a través de la razón de verosimilitud del sistema. La idea que se encuentra detrás de este tipo de modelos es que existen dos decisiones, cada una modelable con un Probit Univariante, pero donde dichas decisiones no son independientes entre sí, pues no se cumple el supuesto de no correlación entre los residuos de cada modelo. Por consiguiente, lo óptimo sería estimar ambas decisiones en forma conjunta.

A diferencia del modelo de ecuaciones lineales simultáneas, en Probit Bivariado si las dos variables dependientes son determinadas conjuntamente, simplemente se pone cada una en el lado derecho de la otra ecuación y luego procedemos como si no hubiera problema de simultaneidad ${ }^{7}$.

El modelo se puede estimar mediante el siguiente sistema de ecuaciones:

$E Q 1:$ Computador $=\beta_{0}+\beta_{1}$ lngastohogar $+\beta_{2}$ niveleducatjefehogar $+\beta_{3}$ mujer $+\beta_{4}$ bonoluz $+\beta_{5}$ rural $+\beta_{6}$ Resd De 6 a 18 años $+\beta_{7}$ Resd. Mayor de 18 años $+\beta_{8}$ TelFijo $+\beta_{9}$ Tel.Movil $+\beta_{10}$ Telecab $+u_{i}$

EQ2: InternetResidencial

$$
\begin{aligned}
& \beta_{0}+\beta_{1} \text { lngastohogar }+\beta_{2} \text { niveleducatjefehogar }+\beta_{3} \text { mujer } \\
& \beta_{4} \text { bonoluz }+\beta_{5} \text { rural } \\
& \beta_{6} \text { Resd De } 6 \text { a } 18 \text { años }+\beta_{7} \text { Resd.Mayor de } 18 \text { años } \\
& \beta_{8} \text { TelFijo }+\beta_{9} \text { Tel.Movil }+\beta_{10} \text { Telecab }+u_{i}
\end{aligned}
$$

Donde:

Ln (gasto del hogar): es la cantidad total de recursos que gasta el

\footnotetext{
${ }^{7}$ Estos modelos permiten la intervención de variables endógenas que pueden presentar varias formas de simultaneidad y causalidad. Por otro lado, resultan muy útiles cuando se estudian variables discretas simultáneamente determinadas. Además, permiten analizar algunos casos en los que variables económicas solo pueden ser definidas en forma discreta.
} 
hogar en compra de bienes y servicios cada mes, expresada en logaritmo natural.

Nivel educativo del jefe de hogar: es el grado académico alcanzado por el jefe de hogar.

Mujer jefa de hogar: variable dummy con valor de 1 para los hogares dirigidos por mujeres.

Bonoluz: variable dummy con valor de 1 para los hogares beneficiarios de este subsidio.

Zona rural: variable dummy con valor de 1 para los hogares establecidos en la zona rural.

Residentes de 6 a 18 años: variable dummy con valor de 1 para los hogares donde residen personas cuyo rango de edad está entre seis y dieciocho años.

Residentes mayores de 18 años: variable dummy con valor de 1 para los hogares donde residen personas mayores de dieciocho años.

Teléfono fijo: variable dummy con valor de 1 para los hogares que disponen de telefonía fija.

Teléfono Móvil: variable dummy con valor de 1 para los hogares donde al menos uno de sus miembros dispone de telefonía móvil.

Telecable: variable dummy con valor de 1 para los hogares que disponen de servicio de difusión por cable.

De acuerdo con los resultados del sistema de ecuaciones, el coeficiente de correlación entre los errores de las ecuaciones es significativo. 
Empleando el test de Wald, para probar la hipótesis de que los errores son independientes, se rechaza la hipótesis nula de independencia debido al valor del chi-cuadrado (X2) de 60.18. (ver tabla 12).

Este resultado sugiere que el modelo Probit Bivariado es más adecuado que la estimación de dos modelos Probit por separado, sin tener pérdidas de eficiencia en la estimación (Greene, 2003).

De acuerdo con los resultados del modelo, el coeficiente del gasto del hogar resulta positivo y significativo tanto para la probabilidad de tener computador en el hogar como para la de disponer del servicio de Internet residencial. Pero, el coeficiente de la disponibilidad de computador es mayor que el del servicio de Internet residencial.

Tabla 12. Probit bivariado para determinantes de COMPUTAdor E INTERnet en HOGARES

\begin{tabular}{|c|c|c|c|c|c|c|c|}
\hline \multirow{2}{*}{ Variables } & \multicolumn{3}{|c|}{ Disp. computador en el hogar } & \multicolumn{3}{|c|}{ Servicio de Internet en hogar } & \multirow{2}{*}{$\begin{array}{c}\text { Efecto } \\
\text { margi- } \\
\text { nal }\end{array}$} \\
\hline & Coef. & d.e. & $\mathrm{Z}$ & Coef. & d.e & $\mathrm{Z}$ & \\
\hline Ln gasto hogar & $.2560^{* * *}$ & .0580 & 4.41 & $.2107^{* * *}$ & 0799 & 2.64 & .0054 \\
\hline Nivel Ed. JH & $.1801^{* * *}$ & .0244 & 7.38 & $.2632^{* * *}$ & .0318 & 8.26 & .0056 \\
\hline Mujer JH & -.0526 & .0511 & -1.03 & -.0596 & .0687 & -0.87 & -.0013 \\
\hline $\begin{array}{l}\text { Subsidio (Bo- } \\
\text { noluz) }\end{array}$ & $-.1976^{* * * *}$ & .0737 & -2.68 & -.0971 & .1104 & -0.88 & -.0029 \\
\hline Rural & $-.1577^{* *}$ & .0665 & 2.37 & $-.3379^{* * *}$ & .1117 & -3.02 & -.0061 \\
\hline De 6 a 18 años & $.1909^{* * * *}$ & .0262 & 7.29 & $.1514^{* * *}$ & .0365 & 4.15 & .0039 \\
\hline $\begin{array}{l}\text { Mayor a } 18 \\
\text { años }\end{array}$ & $.0853^{* * *}$ & .0254 & 3.35 & $.0559^{*}$ & .0325 & 1.72 & 0016 \\
\hline Teléfono fijo & $.7298^{* * *}$ & .0669 & 10.90 & $2.3841^{* * *}$ & .1239 & 19.24 & .1318 \\
\hline Teléfono móvil & $.5354^{* * *}$ & .0926 & 5.78 & $.5783^{* * *}$ & .0990 & 5.84 & .0090 \\
\hline Telecable & $.4547^{* * *}$ & .0627 & 7.26 & $.5603^{* * *}$ & .0853 & 6.57 & .0157 \\
\hline Constante & $-5.018^{* * *}$ & .5406 & -9.28 & $-5.998^{* * *}$ & .7573 & -7.92 & - \\
\hline /athrho & $.4236^{* * *}$ & .0546 & 7.76 & & .3166 & .5307 & - \\
\hline rho & .3999 & .0459 & & & .3064 & .4859 & - \\
\hline
\end{tabular}

Notas. Log-pseudolikelihood $=-1514408.3 ;$ Observaciones $=5,666 ;$ Wald$\operatorname{Chi}^{2}(22)=1151.57 ;$ Prob $>C h i^{2}=0.0000$; Wald-test of rho=0: $\mathrm{Chi}^{2}=60.18^{* * *}$; Prob $>\mathrm{Chi}^{2}=0.0000$. Coef: coeficientes; d.e.: desviación estándar.

${ }^{* * *}$ valor- $p<0.01 ;{ }^{* *}$ valor $-p<0.05 ;{ }^{*}$ valor $-p<0.10$ 
Por otro lado, el nivel educativo del jefe de hogar también es positivo y significativo en ambas ecuaciones, pero el coeficiente es mayor para la probabilidad de Internet residencial que para el computador en el hogar.

Los resultados para los coeficientes que miden la jefatura de hogar por parte de una mujer no resultan significativos para ninguna de las dos ecuaciones. Asimismo, el coeficiente para los hogares que reciben subsidios sociales es negativo y significativo solo para disponibilidad de computador.

Para los hogares establecidos en la zona rural, los coeficientes para la disponibilidad de computador e Internet residencial resultan negativos y significativos. Pero, el coeficiente de disponibilidad de Internet residencial resulta menor que el de computador.

Los coeficientes para los miembros en el hogar resultan positivos y significativos con mayor valor probabilístico para la disponibilidad de computador que para el servicio de Internet residencial. Pero, existe una mayor probabilidad de disponibilidad de computador e Internet en los hogares donde residen miembros cuyas edades oscilan entre seis y dieciocho años.

En cuanto a los servicios finales de telecomunicaciones evaluados, los coeficientes resultaron mayores en el caso de la telefonía residencial, tanto para la probabilidad de tener computador en el hogar como para el Internet residencial.

Esto podría estar explicado por el empaquetamiento de servicios finales de telecomunicaciones, especialmente los paquetes que vinculan a la telefonía fija con el Internet residencial. Sin embargo, la probabilidad de Internet residencial resulta mayor que la de computador en el hogar. Esta diferencia a favor de la probabilidad de disponer de Internet residencial también se evidencia en la telefonía fija y en el telecable. 
No obstante, dada la naturaleza de los datos, el modelo Biprobit no capta completamente el carácter de la correlación (selección) entre las variables estimadas. De hecho, la tenencia de computador determina la posibilidad de conexión a Internet de los hogares que realmente pueden adquirirlo.

Por lo tanto, la muestra de hogares que tienen servicio de Internet residencial no es aleatoria y esta característica de los datos podría generar estimaciones sesgadas (Heckman, 1979).

Heckman desarrolla un modelo con el fin de eliminar las consecuencias del llamado sesgo de selección. Una de las formas más comunes de dicho sesgo es la autoselección. Esta se produce como consecuencia de que los datos que posee el investigador son generados por las decisiones individuales de pertenecer a un grupo $\mathrm{u}$ otro.

En el caso de consumo de bienes o servicios, un individuo primero debe tomar la decisión de participar en determinado mercado y después decidir de qué manera lo hará. Heckman desarrolla un modelo en dos etapas. En una primera etapa, se analiza la decisión de acceso al mercado y, en la segunda, se analiza el consumo.

Su modelo está estructurado de esta manera debido a dos motivos: 1) para poder corregir el sesgo de selección y 2) porque las variables que afectan la decisión de participación no necesariamente son las mismas que determinan el consumo.

La forma de enfrentar este problema, econométricamente hablando, es asumir el modelo Probit Bivariado con una selección de la muestra. Este enfoque adapta el procedimiento de dos pasos de Heckman a este modelo dicotómico (Van de Ven, et al., 1981). Intuitivamente, se aborda el procedimiento Heckman con selección de muestras como un problema de especificación. 
De esta forma, intenta solucionarlo insertando una variable proxy que captura la parte omitida de la muestra truncada que es atribuible a la selección. El Probit Bivariado con modelo de selección de muestras (HeckProbit) se especifica como sigue:

$$
\operatorname{Pr}(\mathrm{Yj}, \mathrm{C}=1)=\Phi[\mathrm{Xj} \theta+\varphi \lambda \mathrm{i}]
$$

Donde $\lambda$ corresponde a la razón inversa de Mill (término de corrección de Heckman). En esta ecuación, la variable dependiente se observa si $\mathrm{Ui}, \mathrm{H}=\mathrm{Xi} \beta \mathrm{H}+\varepsilon \mathrm{i}, \mathrm{H}>0$. Así, la ecuación de disponibilidad o no de computador queda especificada como la ecuación seleccionada.

Empíricamente, el procedimiento sigue los siguientes pasos. En primer lugar, la ecuación de selección se estima por máxima verosimilitud. Entonces, esta estimación se usa para construir la relación inversa de Mill $\left(\lambda=\varphi\left(X^{\prime} i \theta\right) / \Phi\left(X^{\prime} i \theta\right)\right.$ utilizando los pseudo residuos.

Estos pseudo-residuos representan los factores no observados que determinan la decisión del hogar de tener acceso a la computadora. Finalmente, la selección el término de corrección $\lambda$ se incluye en la ecuación Probit de Internet, que también se estima utilizando el procedimiento de máxima verosimilitud.

Heckman estima la segunda etapa por mínimos cuadrados ordinarios (MCO). Si $\lambda$ es significativa en la estimación de la segunda etapa, implicará la existencia de sesgo de selección. En caso contrario, no será necesario realizar la estimación mediante un proceso bietápico, sino que puede ser realizado directamente a través de los modelos clásicos de estimación.

En el modelo estimado en la tabla 13 , la variable $\lambda$ resulta positiva y significativa. Es decir, existe sesgo de autoselección en la muestra de hogares. Esto implica que la adquisición de computadoras es un requisito previo para tener conexión a Internet disponible en el hogar. 
Por tal razón, el modelo bietápico es el más indicado para corregir este problema y obtener estimadores insesgados.

Según los resultados, de los coeficientes que son significativos para ambas ecuaciones, los referentes a la probabilidad de tener computador en el hogar son más altos que los de la disponibilidad de Internet residencial, con excepción de la telefonía fija.

El coeficiente para el gasto del hogar resulta, al igual que en el modelo Probit Bivariado, el de mayor valor tanto para la probabilidad de computador como para la de Internet residencial, seguido del nivel educativo del jefe de hogar.

Para el caso en que una mujer sea la jefa del hogar, el coeficiente resulta negativo y significativo solo para la disponibilidad de computador, al igual que los hogares que reciben subsidios sociales. Estos resultados vedan la posibilidad de que en un hogar dirigido por mujeres y/o reciba subsidios sociales se disponga del servicio de Internet residencial.

El coeficiente que mide la probabilidad de los hogares de la zona rural resulta negativo y significativo para la disponibilidad de Internet residencial. Este resultado es esperable debido al bajo porcentaje de hogares que disponen de computador y de la baja penetración del Internet en la zona urbana.

Para los miembros en el hogar, los coeficientes resultan positivos y significativos, tanto para la disponibilidad de computador como para el servicio de Internet residencial. Estos coeficientes resultan más altos para los hogares donde viven personas cuya edad oscila entre seis y dieciocho años. 
Tabla 13. Modelo de Heckman en dos etapas para computador e Internet en HOGARES

\begin{tabular}{|c|c|c|c|c|c|c|c|}
\hline \multirow[t]{2}{*}{ Variables } & \multicolumn{3}{|c|}{$\begin{array}{l}\text { Disp. computador en el ho- } \\
\text { gar }\end{array}$} & \multicolumn{3}{|c|}{$\begin{array}{l}\text { Servicio de Internet en ho- } \\
\text { gar }\end{array}$} & \multirow[t]{2}{*}{$\begin{array}{l}\text { Efecto } \\
\text { marginal }\end{array}$} \\
\hline & Coef. & d.e. & Z & Coef. & d.e & Z & \\
\hline Ln gasto hogar & $.2952^{* * *}$ & .0468 & 6.30 & $.2509^{* * *}$ & .1172 & 3.14 & $.2952^{* * *}$ \\
\hline Nivel Ed. JH & $.1802^{* * *}$ & .0195 & 9.22 & $.1766^{* * *}$ & .0660 & 2.67 & $.1802^{* * *}$ \\
\hline Mujer JH & $-.0994^{* *}$ & 0487 & -2.04 & -.0430 & .0629 & -0.68 & $-.0994^{* *}$ \\
\hline $\begin{array}{l}\text { Subsidio (Bo- } \\
\text { noluz) }\end{array}$ & $-.1515^{* * *}$ & 0569 & -2.66 & -.1007 & .0851 & -1.18 & $-.1515^{* * *}$ \\
\hline Rural & -.0639 & . 0505 & -1.27 & $-.1401^{* *}$ & .0605 & -2.32 & -.0639 \\
\hline De 6 a 18 años & $.1619^{* * *}$ & .0206 & 7.86 & $.1326^{* *}$ & .0633 & 2.09 & $.1619^{* * *}$ \\
\hline $\begin{array}{l}\text { Mayor a } 18 \\
\text { años }\end{array}$ & $.0889^{* * *}$ & .0190 & 4.68 & $.0683^{* *}$ & .0377 & 2.07 & $.0889^{* * *}$ \\
\hline Teléfono fijo & $.7736^{* * *}$ & .0527 & 14.67 & $1.255^{* * *}$ & .2918 & 4.30 & $.7736^{* * *}$ \\
\hline Teléfono móvil & $.5287^{* * *}$ & .0695 & 7.60 & $.3845^{*}$ & .2149 & 1.79 & $.5287^{* * *}$ \\
\hline Telecable & $.3715^{* * *}$ & .0496 & 7.49 & $.3421^{* *}$ & .1491 & 2.29 & $.3715^{* * *}$ \\
\hline Constante & $-5.337^{* * *}$ & .4402 & -12.13 & $-5.2102^{* *}$ & 2.3774 & -2.19 & $-5.3379^{* * *}$ \\
\hline Mills lambda & $1.0241^{* *}$ & .4960 & 2.06 & & .0519 & 1.9964 & $1.0241^{* *}$ \\
\hline Rho & 1.0000 & & & & & & 1.0000 \\
\hline Sigma & 1.0242 & & & & & & 1.0242 \\
\hline lambda & 1.024 & .4960 & & & & & 1.0242 \\
\hline
\end{tabular}

Notas. Log-pseudolikelihood $=-1514408.3 ;$ Observaciones $=5,666 ;$ Censored Observations $=4728 ;$ Uncensored Observations $=938 ;$ Wald-Chi $^{2}(9)=78.89$; Prob $>\mathrm{Chi}^{2}=0.0000$; Wald-test of rho $=0: \mathrm{Chi}^{2}=60.18^{* * *} ;$ Prob $>\mathrm{Chi}^{2}=0.0000$. Coef: coeficientes; d.e.: desviación estándar.

El coeficiente para la telefonía fija resulta positivo y significativo para ambas ecuaciones, siendo mayor para el Internet residencial que para la disponibilidad de computador. Este resultado muestra la alta incidencia de la telefonía fija en la penetración del Internet residencial a través del empaquetamiento de servicios por parte de los proveedores ${ }^{8}$.

\footnotetext{
${ }^{8}$ La empresa proveedora de servicios de Internet con mayor participación de mercado posee más del $80 \%$ de la red fija disponible en el territorio nacional.
} 
Cabe recordar que la telefonía fija es la tercera red de servicios de mayor cobertura, superada por la telefonía móvil y la difusión por cable. Los coeficientes para la telefonía móvil y el telecable resultan positivos y significativos para ambas estimaciones, siendo mayores para la disponibilidad de computador que para el Internet residencial.

Es necesario hacer la salvedad de que los modelos utilizados para estimar las ecuaciones (Probit Bivariado y Heckprobit) son sensibles a salidas de la normalidad del término de error (ci).

De hecho, la estimación de Heckprobit de $\Phi$ en la ecuación (8) es sensible a la normalidad en $\varepsilon i$, dada la construcción de $\lambda$ i invoca la suposición normal $\left(\Lambda \mathrm{i}=\varphi\left(Z^{\prime} \mathrm{i} \theta\right) / \Phi\left(Z^{\prime} \mathrm{i} \theta\right)\right)$. La no-normalidad en el contexto de las funciones de distribución de probabilidad bivariada es más compleja.

Por tal razón, es mucho más fácil permanecer dentro del marco del procedimiento bietápico. Sin embargo, en ambos modelos los coeficientes relativos al gasto del hogar (como proxy del ingreso) y el nivel educativo del jefe de hogar evidencian altos niveles de significancia estadística. Esto podría implicar que el nivel educativo y el ingreso laboral juegan un papel importante en la decisión de adquirir un computador para el hogar.

Una posible explicación para este resultado es que las personas necesitan algún tipo de experiencia previa para aprovechar plenamente el servicio de Internet residencial. Por lo tanto, el uso de Internet en el trabajo y en la escuela podría aumentar la utilidad de tenerlo en casa y luego la probabilidad de que los hogares adquieran computadores para poder conectarse a la red proveedora de este servicio.

En materia de políticas públicas, este argumento apoyaría la iniciativa del programa República Digital de dotar de computadores, así como de acceso a Internet al interior de los centros educativos a maestros y alumnos de primaria, media y secundaria, debido a que 
esta experiencia previa podría aumentar la utilidad de disponer del servicio en el hogar a través de un proveedor privado.

Estos resultados son consistentes con la investigación de Vicente \& López (2006), quienes encontraron evidencia de que el ingreso y la educación superior son determinantes tanto para la adopción de computadoras, como para el uso de Internet.

Sin embargo, esta relación no se cumpliría para todos los hogares debido a las brechas de acceso territoriales. Los hogares establecidos en áreas urbanas serían más propensos a poseer una computadora y tener servicio de Internet residencial que sus pares en la zona rural.

En tal sentido, Demousis \& Giannakopoulus (2006) evidenciaron que el acceso a Internet depende de variables como el ingreso, la educación, el género, la ubicación geográfica y el costo del servicio.

En línea con estos hallazgos para Latinoamérica, Grazzi y Vergara $\left(2009^{a}\right)$, utilizando un modelo similar al usado en esta investigación, encuentran que tanto el ingreso como la educación y el área geográfica (urbano/rural) son determinantes para la difusión de las TIC. Estos autores consideran que la adopción de computadoras determina completamente la posibilidad del uso de Internet.

\section{Conclusiones y recomendaciones}

Existe una relación positiva entre el gasto del hogar y la probabilidad de tener computador en la casa, así como de disponer del servicio de Internet residencial. Es decir, a mayor gasto del hogar, mayor es la probabilidad de disponer de computador y de servicio de Internet residencial.

El nivel educativo del jefe de hogar también está relacionado positivamente con la probabilidad de tener computador y disponer del servicio de Internet residencial. 
El nivel educativo y el ingreso laboral juegan un papel importante en la decisión de adquirir un computador para el hogar. Una posible explicación para este resultado es que las personas necesitan algún tipo de experiencia previa para aprovechar plenamente el servicio de Internet residencial.

En tal sentido, el uso de Internet en el trabajo y en la escuela podría aumentar la utilidad de tenerlo en casa y luego la probabilidad de que los hogares adquieran computadores para poder conectarse a la red proveedora de este servicio.

En lo concerniente a la educación, la evidencia empírica muestra que la utilización de la banda ancha en el aula, especialmente en la enseñanza secundaria y superior, es un objetivo directamente relacionado con objetivos sociales a largo plazo, en particular, la necesidad vital de tener una población y una mano de obra con dominio de las tecnologías.

En el caso en que una mujer sea la jefa del hogar, la relación es negativa para la disponibilidad de computador al igual que los hogares que reciben subsidios sociales. Estos resultados vedan la posibilidad de que un hogar dirigido por mujeres y/o que reciba subsidios sociales disponga del servicio de Internet residencial.

Para los hogares de la zona rural, existe una relación negativa respecto de la probabilidad de disponer de Internet residencial. Este resultado es esperable debido al bajo porcentaje de hogares que disponen de computador y de la baja penetración del Internet en la zona rural.

Para los miembros en el hogar, los coeficientes resultan positivos y significativos tanto para la disponibilidad de computador como para el servicio de Internet residencial. Estos coeficientes resultan más altos para los hogares donde viven personas cuya edad oscila entre seis y dieciocho años. 
Para los hogares que disponen de telefonía fija, existe una mayor probabilidad de tener Internet residencial y disponibilidad de computador. Este resultado muestra la alta incidencia de la telefonía fija, especialmente en la zona urbana, en la penetración del Internet residencial a través del empaquetamiento de servicios por parte de los proveedores.

Para los hogares cuyos miembros disponen de telefonía móvil y de telecable existe una probabilidad menor que para la telefonía fija para ambas estimaciones, siendo mayores para la disponibilidad de computador que para el Internet residencial.

De acuerdo con los principales hallazgos del estudio, las recomendaciones son las siguientes:

1) Poner en marcha, lo antes posible, la masificación de los servicios de Internet de banda ancha propuestos en el programa República Digital, ya que la asignación que realiza actualmente el mercado es inferior a la socialmente óptima.

2) El programa República Digital, en su eje de acceso, debe incorporar las desigualdades territoriales respecto de los determinantes de servicios finales de telecomunicaciones, con énfasis en el acceso al Internet en su estrategia de inclusión digital.

3) La iniciativa de dotar de computadores a maestros y alumnos, así como de acceso a Internet al interior de los centros educativos, podría aumentar la utilidad de disponer del servicio en el hogar a través de un proveedor privado.

4) La política de desarrollo y masificación del Internet debe priorizar a los hogares de la zona rural, los que reciben subsidios sociales y los dirigidos por mujeres.

5) La principal barrera a la entrada al Internet en el hogar es el computador. Cualquier programa de reducción de brecha que 
quiera tener impacto debe proveer $\mathrm{y} / \mathrm{o}$ financiar a los hogares estos equipos, así como una parte del servicio, priorizando por los de menores ingresos.

6) Es esperable que un hogar que reciba un computador incremente su gasto en telecomunicaciones. Por tal razón, es imprescindible subsidiar por el lado de la oferta-vía fibra oscura de ETED, buscando acuerdos con proveedores de Internet que permitan morigerar el incremento del gasto del hogar.

7) La inclusión digital puede reducir la desigualdad. Las nuevas tecnologías pueden contribuir a que las personas estén mejor integradas en la sociedad, a través de programas amplios dirigidos a quienes tengan necesidades especiales, así como programas específicos para mejorar las destrezas digitales.

\section{Bibliografía}

Agostini, C. A. \& Willington, M. (2010). Radiografía de la brecha digital en Chile: ¿se justifica la intervención del Estado? Estudios Públicos, 199, 5-32.

Demoussis, M. \& Giannakopoulos, N. (2006). The dynamics of home computer ownership in Greece. Information Economics and Policy, 18, 73-86.

Fairlie, R. (2003). Race and the Digital Divide, Berkeley Electronic Journals, Contributions to Economic Analysis and Policy, 3(1), $1-38$.

Fairlie, R. (2004). Is there a Digital Divide? Ethnic and Racial Differences in Access to Technology and Possible Explanation, Final Report to the University of California, Latin Policy Institute \& California Policy Research Institute. 
Fairlie, R. (2006). The Personal Computer and Entrepreneurship, Management Science, 52(2), 187-203.

Gi-Soon \& Song. (2005). The Impact of Information and Communication Technologies (ICTS) on Rural Households: A Holistic Approach Applied to the Case of Lao People's Democratic Republic. Jakarta: UNV/UNDP.

Gómez, F., Jaqueline, M. Cruz, O. \& Mariano, J. (2015). Impacto de la crisis eléctrica en República Dominicana: informe sobre hogares. Santo Domingo: INTEC-BID.

Goolsbee \& Klenow. (2002). Evidence on learning and network externalities in the diffusion of home computers. Journal of Law and Economics, Vol 45(2): 317-343.

Grazzi, M. \& Vergara, S. (2008). What Drives ICT diffusion in Developing Countries? Evidence from Paraguay, Working Paper presented at LACEA 2009, Rio de Janeiro, November 23.

Grazzi, M. \& Vergara, S. (2009). ICT Access in Latin America: Evidence from Household Level. Division of Production, Productivity and Management. (Mimeo) UN-ECLAC.

Grazzi, M. \& Vergara, S. (2011). ITC in Latin America. Evidence from household level. Division of Production, Productivity and Management. Economic Commission for Latin America and the Caribbean (ECLAC) United Nations.

Greene, W. (1996). Marginal effects in the bivariate probit model. Working paper number 96-11. Department of Economics, Stern School of Business, New York University.

Greene, W. (1997). Econometric Analysis (3rd ed.). Englewood Cliffs, NJ: Prentice Hall. 
Greenstein \& Prince. (2006). The Diffusion of the Internet and the Geography of the Digital Divide in the United States. NBER. Working Paper 12182. National Bureau of Economic Research.

Gutierrez, L. H., \& Gamboa, L. F. (2008). An approximation to the digital divide among low income people in Colombia, Mexico and Peru: two composite indexes. Serie documentos de trabajo, No. 39. Universidad del Rosario.

Hausman, J. \& Wise, D. A. (1978). A Conditional Probit Model for Qualitative Choice: Discrete Decisions Recognizing Interdependence and Heterogeneous Preferences. Econometrica, 46, 403-426.

Heckman, J. (1979). Sample Selection Bias as a Specification Error, Econometrica, 47(1), 153-161.

Hilbert, M. (2011). The end justifies the definition: The manifold outlooks on the digital divide and their practical usefulness for policy-making. Telecommunications Policy, 35(8), 715-736.

Katz, R. (2012). Impact of Broadband on the Economy. Ginebra: UIT.

Katz, R. L. (2009). La contribución de las tecnologías de la información y las comunicaciones al desarrollo económico: propuestas de América Latina a los retos económicos actuales. Madrid: ARIEL.

Maddala, G. (1983). Limited dependent and qualitative variables in econometrics. Cambridge: Cambridge University Press.

McFadden, D. (1981). Econometric Models of Probabilistic Choice. In C.F. Manski \& D. McFadden (eds.), Structural Analysis of Discrete Data with Econometric Applications. Cambridge, Massachusetts: MIT Press. 
McFadden, D. (1984). Econometric Analysis of Qualitative Response Models. En Griliches, Z. \& Intriligator, M. D. (eds.): Handbook of Econometrics, Vol. II, New York: Elsevier Science Publishers BV.

McFadden, D. \& Train, D. (2000). Mixed MNL Models for Discrete Response. Journal of Applied Econometrics, 15, 447-470.

Murphy, K. \& Topel, R. (1985). Estimation and inference in two step econometric models. Journal of Business and Economic Statistics, 3, 370-379.

Ono, H. \& Zavodny, M. (2002). Gender and the Internet, Working Paper Series, Federal Reserve Bank of Atlanta, No. 2002-10.

Ono, H. \& Zavodny, M. (2007a). Immigrants, English Ability and the Digital Divide, IZA Working Paper N. 3124.

Ono, H. \& Zavodny, M. (2007b). Digital Inequality: A Five Country Comparison using Microdata, Social Science Research 36-3 (2007), 1135-1155.

OSILAC. (2007). Characteristics of Households with ICTs in Latin America and the Caribbean, Division of Production, Productivity and Management, ECLAC, United Nations, December.

Peres, W., \& Hilbert, M. (2009). La sociedad de la información en América Latina. Santiago de Chile: CEPAL.

Rivers, D. \& Vuong, Q. (1987). Limited information estimators and exogeneity tests for simultaneous probit models. Journal of Econometrics, 39, 347-366.

Toudert, D. $\left(2013^{\mathrm{a}}\right)$. La brecha digital en los contextos de marginación socioterritorial de las localidades mexicanas de más de 
2500 habitantes: Exploración y discusión. Comunicación y Sociedad, 19, 53-180.

Trung, Tran Quoc. (2007). Trade Liberalization and Development in ICT Sector and its impact on household welfare in Viet Nam. Asia-Pacific Research and Training Network on Trade. s.1.: Working Paper Series, No. 33, 2007.

Van de Ven, W.P.M. \& Van Praag, B. M. (1981). The Demand for Deductibles in Private Health Insurance: A Probit Model with Sample Selection, Journal of Econometrics, 17, 229-252.

Venkatesh, V. \& Shih, C. (2005). An Investigation of theories of diffusion in the Global Context: A comparative Study of the US, Sweden and India, Center for Research Technology, University of California, Irvine.

Venkatesh, V. \& Brown, S. A. (2001). A Longitudinal Investigation of Personal Computers in Homes: Adoption Determinants and Emerging Challenges, MIS Quarterly, 25(1), 71-102.

Vicente, M.R. \& López, A. J. (2006). Patterns of ICT Diffusion across the European Union, Economic Letters, 93, 45-51. 